\title{
CONCENTRATION OF THE COMPLEXITY OF SPHERICAL PURE $p$-SPIN MODELS AT ARBITRARY ENERGIES
}

\author{
ELIRAN SUBAG AND OFER ZEITOUNI
}

\begin{abstract}
We consider critical points of the spherical pure $p$-spin spin glass model with Hamiltonian $H_{N}(\boldsymbol{\sigma})=$ $\frac{1}{N^{(p-1) / 2}} \sum_{i_{1}, \ldots, i_{p}=1}^{N} J_{i_{1}, \ldots, i_{p}} \sigma_{i_{1}} \cdots \sigma_{i_{p}}$, where $\boldsymbol{\sigma}=\left(\sigma_{1}, \ldots, \sigma_{N}\right) \in \mathbb{S}^{N-1}:=\left\{\boldsymbol{\sigma} \in \mathbb{R}^{N}:\|\boldsymbol{\sigma}\|_{2}=\sqrt{N}\right\}$ and $J_{i_{1}, \ldots, i_{p}}$ are i.i.d standard normal variables. Using a second moment analysis, we prove that for $p \geq 32$ and any $E>-E_{\star}$, where $E_{\star}$ is the (normalized) ground state, the ratio of the number of critical points $\sigma$ with $H_{N}(\sigma) \leq N E$ and its expectation asymptotically concentrates at 1 . This extends to arbitrary $E$ a similar conclusion of [Sub17a].
\end{abstract}

\section{INTRODUCTION}

The Hamiltonian of the spherical pure $p$-spin spin glass model is given by

$$
H_{N}(\boldsymbol{\sigma}):=\frac{1}{N^{(p-1) / 2}} \sum_{i_{1}, \ldots, i_{p}=1}^{N} J_{i_{1}, \ldots, i_{p}} \sigma_{i_{1}} \cdots \sigma_{i_{p}},
$$

where $\boldsymbol{\sigma}=\left(\sigma_{1}, \ldots, \sigma_{N}\right)$ belongs to the sphere $\mathbb{S}^{N-1}:=\left\{\boldsymbol{\sigma} \in \mathbb{R}^{N}:\|\boldsymbol{\sigma}\|_{2}=\sqrt{N}\right\}$, and $J_{i_{1}, \ldots, i_{p}}$ are i.i.d standard normal variables. The model was introduced by Crisanti and Sommers CS92 for general $p \geq 2$ as a continuous variant of the same models with Ising spins, while for $p=2$ it was already considered by Kosterlitz, Thouless and Jones KTJ76 in analogy to the Sherrington-Kirkpatrick model SK75.

For any subset $B \subset \mathbb{R}$, let $\operatorname{Crt}_{N}(B)$ be the number of critical points of $H_{N}$, at which it attains a value in $N B=\{N x: x \in B\}$,

$$
\operatorname{Crt}_{N}(B):=\#\left\{\boldsymbol{\sigma} \in \mathbb{S}^{N-1}: \nabla H_{N}(\boldsymbol{\sigma})=0, H_{N}(\boldsymbol{\sigma}) \in N B\right\},
$$

where $\nabla H_{N}(\boldsymbol{\sigma})$ denotes the spherical gradient of $H_{N}(\boldsymbol{\sigma})$. We refer to $\operatorname{Crt}_{N}((-\infty, u))$ as the complexity of $H_{N}$ at energies below $N u$. In their seminal work [ABAČ13], Auffinger, Ben Arous, and Černý proved that

$$
\lim _{N \rightarrow \infty} \frac{1}{N} \log \mathbb{E C r t}_{N}((-\infty, u))=\Theta_{p}(u)
$$

for

$$
\Theta_{p}(u)= \begin{cases}\frac{1}{2} \log (p-1)-\frac{p-2}{4(p-1)} u^{2}-J(u) & \text { if } u \leq-E_{\infty}, \\ \frac{1}{2} \log (p-1)-\frac{p-2}{4(p-1)} u^{2} & \text { if }-E_{\infty} \leq u \leq 0, \\ \frac{1}{2} \log (p-1) & \text { if } u \geq 0,\end{cases}
$$

where $E_{\infty}=E_{\infty}(p)=2 \sqrt{\frac{p-1}{p}}$ and for $u \leq-E_{\infty}$

$$
J(u)=-\frac{u}{E_{\infty}^{2}} \sqrt{u^{2}-E_{\infty}^{2}}-\log \left(-u+\sqrt{u^{2}-E_{\infty}^{2}}\right)+\log E_{\infty} .
$$

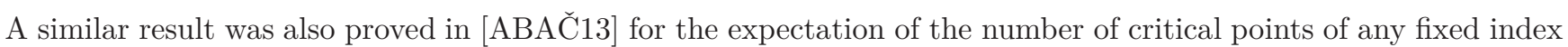
$k \geq 0$. In particular, it was shown that for any $k \geq 0$ and $\epsilon>0$, with probability going to 1 as $N \rightarrow \infty$, there are no critical points $\boldsymbol{\sigma}$ of index $k$ with $H_{N}(\boldsymbol{\sigma}) \in N\left(-E_{\infty}+\epsilon, \infty\right)$.

Define the ground-state energy

$$
-E_{\star}:=\lim _{N \rightarrow \infty} \frac{1}{N} \mathbb{E} \min _{\boldsymbol{\sigma} \in \mathbb{S}^{N-1}} H_{N}(\boldsymbol{\sigma}) .
$$

This project has received funding from the European Research Council (ERC) under the European Union's Horizon 2020 research and innovation programme (grant agreement No. 692452) and from the Israel Science Foundation (grant agreement No. 2055/21). 
The value of $E_{\star}$ can be computed from the Parisi formula [Tal06, Che13] for the free energy by letting the temperature go to zero or directly from its zero temperature analogue [CS17, JT17]; from the TAP representation [Sub18, Sub21] for the free energy; or from the analysis of critical points, as we will now explain. Define $E_{0}=E_{0}(p)>0$ as the unique value such that $\Theta_{p}\left(-E_{0}\right)=0$. Then, by Markov's inequality, $E_{\star} \leq E_{0}$. In fact, it was proved in $\mathrm{ABAC \check {13 }}$ that $E_{\star}=E_{0}$ using the Parisi formula, whose solution in the pure case has 1 step of replica symmetry breaking. Another way to prove the matching bound $E_{\star} \geq E_{0}$ is to show that $\operatorname{Crt}_{N}((-\infty, u))$ concentrates around its mean for levels $u$ in some right neighborhood of $-E_{0}$. This was proved in Sub17a] by a second moment argument, independently of the Parisi formula.

Our main result is the following asymptotic matching of the second moment and first moment squared of $\operatorname{Crt}_{N}((-\infty, u))$, for large enough $p$ and all energies $u>-E_{\infty}$.

Theorem 1. For any $p \geq 32$ and $u \in\left(-E_{\infty}, \infty\right)$,

$$
\lim _{N \rightarrow \infty} \frac{\mathbb{E}\left\{\left(\operatorname{Crt}_{N}((-\infty, u))\right)^{2}\right\}}{\left(\mathbb{E}\left\{\operatorname{Crt}_{N}((-\infty, u))\right\}\right)^{2}}=1 .
$$

Consequently, in $L^{2}$ and in probability,

$$
\frac{\operatorname{Crt}_{N}((-\infty, u))}{\mathbb{E C r t}_{N}((-\infty, u))} \stackrel{N \rightarrow \infty}{\longrightarrow} 1
$$

The same result was proved in Sub17a for any $u \in\left(-E_{\star},-E_{\infty}\right)$ and general $p \geq 3$. Moreover, it was proved there that for any $u \in\left(-E_{\star}, \infty\right)$ and $p \geq 3$, the moments match at exponential scale, i.e., $\frac{1}{N} \log$ of the ratio in (1.3) goes to 0 , a fact that will be used in our proof of Theorem 11. The value $-E_{\infty}$ represents a threshold beyond which a certain shift (corresponding to $U$, see (2.1)) enters the bulk of the spectrum of a certain GOE matrix $X_{N-1}$ related to the Hessian of $H_{N}(\boldsymbol{\sigma})$; compared with [Sub17a], the latter fact requires a more delicate analysis of the asymptotics of certain determinants appearing in the computation of second moments, see (2.2). The restriction to $p \geq 32$ is needed in our analysis of the latter asymptotics. We expect that Theorem 1 remains true for $p \geq 3$, but we do not have a proof for that; while our methods probably can be refined to yield a lower threshold than 32, it is clear that additional ingredients are needed to lower the threshold to 3. In particular, the use of Hölder's inequality in one step of the proof, see (2.22), would need to be replaced by directly evaluating determinants of correlated matrices, on a rare event.

Combining (1.4) with Sub17a, Corollary 2] and (1.2), we obtain the following.

Corollary 2. For any $p \geq 32$ and $u \in\left(-E_{\star}, \infty\right)$,

$$
\frac{1}{N} \log \operatorname{Crt}_{N}((-\infty, u)) \stackrel{N \rightarrow \infty}{\longrightarrow} \Theta_{p}(u), \quad \text { in probability }
$$

Before we turn to the proofs, we mention earlier related works concerning the critical points of spin glass models. We start with the pure spherical models which we consider in the current work. Their mean complexity was first studied in physics in the context of the TAP approach, see for example the works of Crisanti and Sommers [CS95] and Crisnati, Leuzzi and Rizzo [CLR03. Fyodorov [Fyo04 and Fyodorov and Williams [FW07] also studied the mean complexity of a closely related model, using the Kac-Rice formula. In the rigororus mathematical literature, the complexity was first studied in the seminal work of Auffinger, Ben Arous, and Černý ABAČ13. As mentioned above, similarly to (1.2), they also computed the mean complexity of critical points of a given index. Auffinger and Gold AG20 recently claimed that the second moment and first moment squared of the complexity of critical points of any fixed index match at exponential scale, for the relevant energies below $-E_{\infty}$. In [SZ17] the authors studied the extremal point process formed by critical values in the vicinity of the global minimum of $H_{N}(\boldsymbol{\sigma})$ and showed that, when properly centered, it converges to a Poisson process of exponential intensity. The connection between the same critical points and the Gibbs measure at sufficiently low temperature was studied in Sub17b. In particular, it was shown there that the Gibss measure concentrates on spherical bands of temperature dependent radius around few of the deepest critical points. The relevance of critical points with values macroscopically larger than the ground state energy to the free energy, at temperatures near the critical temperature, was recently studied by Ben Arous and Jagannath BAJ21.

Moving to the mixed spherical models, Auffinger and Ben Arous ABA13 computed the mean complexity for general mixtures. As in [ABAČ13, their results also cover the mean complexity of critical points of a given index. 
Matching of the second moment and first moment squared of the complexity at exponential scale was proved by the authors and Ben Arous in BASZ20, for mixed models sufficiently close to a pure model in an appropriate sense, and energies close to the ground state. For the same models and at low temperature, some analogues of the results on the Gibbs measure from Sub17b] were obtained in BASZ20. In contrast to the pure models, for the mixed models the Gibbs measure was shown there to concentrate on bands around points which approximately maximize the restriction of $H_{N}(\boldsymbol{\sigma})$ to a sphere of radius $\sqrt{N q}$, for appropriate $q \in(0,1)$. For general mixed spherical models at arbitrary sub-critical temperature the same was proved in [Sub18]. In fact, when adding an appropriate TAP correction, the same even holds for general mixed models with Ising spins, see the works of Chen, Panchenko and the first author [CPS, CPS20].

For the bipartite spherical mixed models, bounds on the mean complexity were computed by Auffinger and Chen AC14. Recently, McKenna McK21 computed the exact asymptotics of the complexity, building on a more general framework developed by him, Ben Arous and Bourgade in BABM21a, BABM21b. Finally, Kivimae Kiv21 proved the asymptotic matching of second moment and first moment squared of the complexity at exponential scale for the pure bipartite models with large enough number of spins and at energies sufficiently close to the ground state.

\section{Proofs}

The overlap of two points $\boldsymbol{\sigma}$ and $\boldsymbol{\sigma}^{\prime}$ is defined as $R\left(\boldsymbol{\sigma}, \boldsymbol{\sigma}^{\prime}\right):=\frac{1}{N} \boldsymbol{\sigma} \cdot \boldsymbol{\sigma}^{\prime}$. For any subset $I_{R} \subset[-1,1]$, we define

$$
\begin{aligned}
{\left[\operatorname{Crt}_{N}\left(B, I_{R}\right)\right]_{2}:=\#\left\{\left(\boldsymbol{\sigma}, \boldsymbol{\sigma}^{\prime}\right) \in\left(\mathbb{S}^{N-1}\right)^{2}: R\left(\boldsymbol{\sigma}, \boldsymbol{\sigma}^{\prime}\right) \in I_{R},\right.} \\
\left.\quad \nabla H_{N}(\boldsymbol{\sigma})=\nabla H_{N}\left(\boldsymbol{\sigma}^{\prime}\right)=0, H_{N}(\boldsymbol{\sigma}), H_{N}\left(\boldsymbol{\sigma}^{\prime}\right) \in N B\right\} .
\end{aligned}
$$

That is, $\left[\operatorname{Crt}_{N}\left(B, I_{R}\right)\right]_{2}$ is the number of pairs of critical points $\left(\boldsymbol{\sigma}, \boldsymbol{\sigma}^{\prime}\right)$ with overlap in $I_{R}$ and values in $N B$. Note that $\mathbb{E}\left[\operatorname{Crt}_{N}(B,[-1,1])\right]_{2}$ is the second moment of $\operatorname{Crt}_{N}(B)$.

Our proof starts from the integral formulas for $\mathbb{E C r t}_{N}(B)$ and $\mathbb{E}\left[\operatorname{Crt}_{N}\left(B, I_{R}\right)\right]_{2}$ derived in $\underline{\mathrm{ABAC} 13}$ and Sub17a, using the Kac-Rice formula, which we recall in Section 2.1. When we substitute the latter formulas to (1.3), the proof of Theorem 1 reduces to establishing certain upper bounds for large $N$ on the corresponding integrands, which depend on an overlap value in $[-1,1]$. These bounds will be proved in Sections $2.2[2.4$ for different ranges of the overlap. In Section 2.5 we will combine those bounds and complete the proof of Theorem 1 Lastly, in Section 2.6 we will prove Corollary 2 .

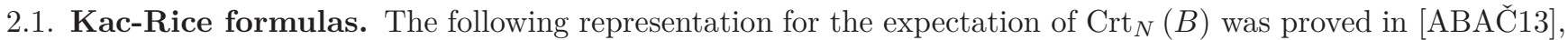
using the Kac-Rice formula (for the latter, see e.g. AT07, Theorem 12.1.1]). Throughout the paper, the normalization we use for a GOE matrix of dimension $N \times N$ is that the variance is $1 / N$ off-diagonal and $2 / N$ on-diagonal. We denote by $I$ the identity matrix.

Lemma 3. ABAČ13, Lemmas 3.1, 3.2] For any $p \geq 3$ and any interval $B \subset \mathbb{R}$,

$$
\mathbb{E}\left\{\operatorname{Crt}_{N}(B)\right\}=\omega_{N}\left(\frac{p-1}{2 \pi}(N-1)\right)^{\frac{N-1}{2}} \mathbb{E}\left\{\left|\operatorname{det}\left(\mathbf{X}_{N-1}-\sqrt{\frac{1}{N-1} \frac{p}{p-1}} U I\right)\right| \mathbf{1}\{U \in \sqrt{N} B\}\right\},
$$

where $\mathbf{X}_{N-1}$ is a GOE matrix of dimension $N-1 \times N-1$ independent of $U \sim N(0,1)$, and

$$
\omega_{N}:=\frac{2 \pi^{N / 2}}{\Gamma(N / 2)}
$$

is the surface area of the $N-1$-dimensional unit sphere.

In Section $\mathrm{A}$ we will define the two pairs $\left(\mathbf{X}_{N-1}^{(i)}(r)\right)_{i=1}^{2}$ and $\left(\mathbf{E}_{N-1}^{(i)}\left(r, u_{1}, u_{2}\right)\right)_{i=1}^{2}$ of $N-1 \times N-1$ matrices, for any $r \in(-1,1)$ and $u_{1}, u_{2} \in \mathbb{R}$. The random matrices $\mathbf{X}_{N-1}^{(i)}(r)$ are correlated, and the upper-left $N-2 \times$ $N-2$ submatrix of each of them has the same distribution as the corresponding submatrix of a GOE matrix. The deterministic matrices $\mathbf{E}_{N-1}^{(i)}\left(r, u_{1}, u_{2}\right)$ are zero everywhere except for the one element in the right-bottom corner. Let $\left(U_{1}(r), U_{2}(r)\right) \sim N\left(0, \Sigma_{U}(r)\right)$ be two Gaussian variables with covariance matrix $\Sigma_{U}(r)$ given by (A.4), independent of the latter matrices. The following representation for the expectation of $\left[\operatorname{Crt}_{N}\left(B, I_{R}\right)\right]_{2}$ was proved in Sub17a]. 
Lemma 4. Sub17a, Lemma 4] For any $p \geq 3$ and any intervals $B \subset \mathbb{R}$ and $I_{R} \subset(-1,1)$,

$$
\begin{aligned}
& \mathbb{E}\left\{\left[\operatorname{Crt}_{N}\left(B, I_{R}\right)\right]_{2}\right\}=C_{N} \int_{I_{R}} d r(\mathcal{G}(r))^{N} \mathcal{F}(r) \\
& \times \mathbb{E}\left\{\prod_{i=1,2}\left|\operatorname{det}\left(\mathbf{X}_{N-1}^{(i)}(r)-\sqrt{\frac{1}{N-1} \frac{p}{p-1}} U_{i}(r) I+\mathbf{E}_{N-1}^{(i)}\left(r, U_{1}(r), U_{2}(r)\right)\right)\right| \cdot \mathbf{1}\left\{U_{1}(r), U_{2}(r) \in \sqrt{N} B\right\}\right\},
\end{aligned}
$$

where

$$
\begin{gathered}
C_{N}=\omega_{N} \omega_{N-1}\left(\frac{(N-1)(p-1)}{2 \pi}\right)^{N-1}, \mathcal{G}(r)=\left(\frac{1-r^{2}}{1-r^{2 p-2}}\right)^{\frac{1}{2}}, \\
\mathcal{F}(r)=(\mathcal{G}(r))^{-3}\left(1-r^{2 p-2}\right)^{-\frac{1}{2}}\left(1-\left(p r^{p}-(p-1) r^{p-2}\right)^{2}\right)^{-\frac{1}{2}} .
\end{gathered}
$$

For small overlaps $r$, we have the following.

Corollary 5. For any $p \geq 3$ and constant $T>0$, there exists a function $B(r)=O\left(r^{3}\right)$, which may depend on $T$, such that the following holds. For any $a_{N}, b_{N} \in[-T, T]$ and interval $I_{N}=\left(r_{N}, r_{N}^{\prime}\right)$ such that $r_{N}, r_{N}^{\prime} \rightarrow 0$,

$$
\limsup _{N \rightarrow \infty} \frac{\mathbb{E}\left\{\left[\operatorname{Crt}_{N}\left(\left(a_{N}, b_{N}\right), I_{N}\right)\right]_{2}\right\}}{\left(\mathbb{E}\left\{\operatorname{Crt}_{N}\left(\left(a_{N}, b_{N}\right)\right)\right\}\right)^{2}} \leq \limsup _{N \rightarrow \infty} \sup _{u_{1}, u_{2} \in\left(a_{N}, b_{N}\right)} \int_{I_{N}} \sqrt{\frac{N}{2 \pi}} e^{-\frac{1}{2} N r^{2}+N B(r)} \Delta_{N}\left(r, u_{1}, u_{2}\right) d r,
$$

where

$$
\Delta_{N}\left(r, u_{1}, u_{2}\right):=\frac{\mathbb{E}\left\{\prod_{i=1,2}\left|\operatorname{det}\left(\mathbf{X}_{N-1}^{(i)}(r)-\sqrt{\frac{N}{N-1} \frac{p}{p-1}} u_{i} I+\mathbf{E}_{N-1}^{(i)}\left(r, \sqrt{N} u_{1}, \sqrt{N} u_{2}\right)\right)\right|\right\}}{\prod_{i=1,2} \mathbb{E}\left\{\left|\operatorname{det}\left(\mathbf{X}_{N-1}-\sqrt{\frac{N}{N-1} \frac{p}{p-1}} u_{i} I\right)\right|\right\}},
$$

and $\mathbf{X}_{N-1}$ is distributed as in Lemma 3

Proof. The corollary follows from Lemmas 3 and 4 since as $r \rightarrow 0, \mathcal{F}(r)=1+O(r), \mathcal{G}(r)=e^{-\frac{1}{2} r^{2}+O\left(r^{4}\right)}$ and uniformly in $r \in I_{N}$ and $u_{1}, u_{2} \in\left(a_{N}, b_{N}\right)$,

and since

$$
\begin{aligned}
\varphi_{\Sigma_{U}(r)}\left(u_{1}, u_{2}\right) & :=\frac{1}{2 \pi}\left(\operatorname{det}\left(\Sigma_{U}(r)\right)\right)^{-1 / 2} \exp \left\{-\frac{1}{2}\left(u_{1}, u_{2}\right)\left(\Sigma_{U}(r)\right)^{-1}\left(u_{1}, u_{2}\right)^{T}\right\} \\
& =\left(1+O\left(r^{p}\right)\right) \frac{1}{2 \pi} \exp \left\{-\frac{1}{2}\left(u_{1}^{2}+u_{2}^{2}\right)+\left(u_{1}+u_{2}\right)^{2} O\left(r^{p}\right)\right\},
\end{aligned}
$$

$$
\lim _{N \rightarrow \infty} \frac{C_{N}}{\omega_{N}^{2}\left(\frac{p-1}{2 \pi}(N-1)\right)^{N-1}} / \sqrt{\frac{N}{2 \pi}}=\lim _{N \rightarrow \infty} \frac{\omega_{N-1}}{\omega_{N}} / \sqrt{\frac{N}{2 \pi}}=1 .
$$

2.2. Large overlaps and energies. The following lemma follows from the asymptotics at exponential scale derived

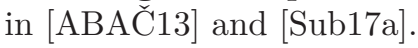

Lemma 6. For any $p \geq 3$ and $u \in\left(-E_{\infty}, \infty\right)$, there exist some sequences of real numbers $a_{N}, b_{N} \rightarrow \min \{u, 0\}$ and $\rho_{N} \rightarrow 0$, such that

$$
\lim _{N \rightarrow \infty} \frac{\mathbb{E}\left\{\left(\operatorname{Crt}_{N}((-\infty, u))\right)^{2}\right\}}{\left(\mathbb{E}\left\{\operatorname{Crt}_{N}((-\infty, u))\right\}\right)^{2}} / \frac{\mathbb{E}\left\{\left[\operatorname{Crt}_{N}\left(\left(a_{N}, b_{N}\right),\left(-\rho_{N}, \rho_{N}\right)\right)\right]_{2}\right\}}{\left(\mathbb{E}\left\{\operatorname{Crt}_{N}\left(\left(a_{N}, b_{N}\right)\right)\right\}\right)^{2}}=1 .
$$

Proof. Let $\rho \in(0,1)$ and $\epsilon>0$. To prove the lemma it is enough to show that if $u<0$ then

$$
\begin{aligned}
\lim _{N \rightarrow \infty} \mathbb{E}\left\{\left[\operatorname{Crt}_{N}((u-\epsilon, u),(-\rho, \rho))\right]_{2}\right\} / \mathbb{E}\left\{\left(\operatorname{Crt}_{N}((-\infty, u))\right)^{2}\right\} & =1, \\
\lim _{N \rightarrow \infty} \mathbb{E}\left\{\operatorname{Crt}_{N}((u-\epsilon, u))\right\} / \mathbb{E}\left\{\operatorname{Crt}_{N}((-\infty, u))\right\} & =1,
\end{aligned}
$$


and if $u \geq 0$ then

$$
\begin{array}{r}
\lim _{N \rightarrow \infty} \mathbb{E}\left\{\left[\operatorname{Crt}_{N}((-\epsilon, \epsilon),(-\rho, \rho))\right]_{2}\right\} / \mathbb{E}\left\{\left(\operatorname{Crt}_{N}((-\infty, u))\right)^{2}\right\}=1, \\
\lim _{N \rightarrow \infty} \mathbb{E}\left\{\operatorname{Crt}_{N}((-\epsilon, \epsilon))\right\} / \mathbb{E}\left\{\operatorname{Crt}_{N}((-\infty, u))\right\}=1 .
\end{array}
$$

The equalities (2.7) and (2.9) follow from Theorem 2.8 of ABAČ13. For $u \in\left(-E_{\star},-E_{\infty}\right)$, (2.6) was proved in Lemma 20 of Sub17a]. The proof of (2.6) and (2.8) (for $u>-E_{\infty}$ ) follows by the same argument if one is able to extend Part (i) of Lemma 6 of Sub17a], used in the proof of Lemma 20 there, to subsets $B \subset\left(-E_{\infty}, E_{\infty}\right)$ instead of subsets $B \subset\left(-\infty,-E_{\infty}\right)$ as in the original statement of the lemma. The proof of the latter generalizes easily if one shows that for any $r \in(-1,1), g_{r}\left(u_{1}, u_{2}\right)$ is strictly concave on $\left(-E_{\infty}, E_{\infty}\right)^{2}$ where

$$
g_{r}\left(u_{1}, u_{2}\right)=-\frac{1}{2}\left(u_{1}, u_{2}\right)\left(\Sigma_{U}(r)\right)^{-1}\left(\begin{array}{l}
u_{1} \\
u_{2}
\end{array}\right)+\Omega\left(\sqrt{\frac{p}{p-1}} u_{1}\right)+\Omega\left(\sqrt{\frac{p}{p-1}} u_{2}\right)
$$

and

$$
\Omega(x)= \begin{cases}\frac{x^{2}}{4}-\frac{1}{2} & \text { if }|x| \leq 2 \\ \frac{x^{2}}{4}-\frac{1}{2}-\frac{|x|}{2} \sqrt{\frac{x^{2}}{4}-1}+\log \left(\sqrt{\frac{x^{2}}{4}-1}+\frac{|x|}{2}\right) & \text { if }|x|>2 .\end{cases}
$$

The concavity of $g_{r}\left(u_{1}, u_{2}\right)$ was proved in Sub17a for $\left(u_{1}, u_{2}\right) \in\left(-\infty,-E_{\infty}\right)^{2}$, we complete the current proof by proving concavity for $\left(u_{1}, u_{2}\right) \in\left(-E_{\infty}, E_{\infty}\right)^{2}$. For such $\left(u_{1}, u_{2}\right) \in\left(-E_{\infty}, E_{\infty}\right)^{2}$, the Hessian of $g_{r}\left(u_{1}, u_{2}\right)$ equals

$$
-\left(\Sigma_{U}(r)\right)^{-1}+\left(\begin{array}{cc}
\frac{1}{2} \frac{p}{p-1} & 0 \\
0 & \frac{1}{2} \frac{p}{p-1}
\end{array}\right)
$$

since for $x \in\left(-E_{\infty}, E_{\infty}\right)$,

$$
\frac{d^{2}}{d x^{2}}\left(\Omega\left(\sqrt{\frac{p}{p-1}} x\right)\right)=\frac{1}{2} \frac{p}{p-1} .
$$

Hence, if we show that the eigenvalue: 1 of $\Sigma_{U}(r)$ are less than $2(p-1) / p$, it will follow that $g_{r}\left(u_{1}, u_{2}\right)$ is strictly concave on $\left(-E_{\infty}, E_{\infty}\right)^{2}$.

The eigenvalues of $\Sigma_{U}(r)$ are equal to $\Sigma_{U, 11}(r) \pm \Sigma_{U, 12}(r)$. One can verify that

$$
\Sigma_{U, 11}(r) \pm \Sigma_{U, 12}(r)=\frac{1-r^{2 p-2} \pm(p-1) r^{p-2}\left(1-r^{2}\right)}{1 \mp\left(p r^{p}-(p-1) r^{p-2}\right)},
$$

which are less than $2(p-1) / p$ if

$$
h(r):=p-2+p r^{2 p-2} \pm(p-2)(p-1) r^{p-2} \mp(p-1) p r^{p}>0 .
$$

Since $h(0)>0$ and $h( \pm 1) \geq 0$, to prove that $h(r)>0$ for $r \in(-1,1)$ it will be enough to show the same for any critical point $r$ of $h(r)$. Since

$$
h^{\prime}(r)=\frac{1}{r}(2 p-2)\left[p r^{2 p-2} \pm \frac{1}{2}(p-2)^{2} r^{p-2} \mp \frac{1}{2} p^{2} r^{p}\right],
$$

at a critical point

$$
p r^{2 p-2}=\mp \frac{1}{2}(p-2)^{2} r^{p-2} \pm \frac{1}{2} p^{2} r^{p}
$$

and

$$
h(r)=p-2 \pm \frac{1}{2} p(p-2) r^{p-2}\left(1-r^{2}\right) .
$$

Since $\left|r^{p-2}\left(1-r^{2}\right)\right|<2 / p$ for all $r \in(-1,1)$, we conclude (2.10) and that $g_{r}\left(u_{1}, u_{2}\right)$ is strictly concave on $\left(-E_{\infty}, E_{\infty}\right)^{2}$.

\footnotetext{
${ }^{1}$ The eigenvalues of $\Sigma_{U}(r)$ are positive for any $r \in(-1,1)$, see Remark 31 in Sub17a].
} 
2.3. Intermediate overlaps. Lemma 6 allows us to restrict to small overlap values $r \in\left(-\rho_{N}, \rho_{N}\right)$, but with no specific bound on the rate at which $\rho_{N} \rightarrow 0$. In this section we prove that for appropriate $C>0$, overlaps $r$ with $|r| \geq C \sqrt{\log N / N}$ are negligible as $N \rightarrow \infty$.

Lemma 7. For any $p \geq 3$ and constant $T \in\left(0, E_{\infty}\right)$, the following holds, with the function $B(r)=O\left(r^{3}\right)$ from Corollary [5. For any sequence $\rho_{N} \rightarrow 0$, setting

$$
I_{N}:=\left(-\rho_{N}, \rho_{N}\right) \backslash(-C \sqrt{\log N / N}, C \sqrt{\log N / N}),
$$

for some $C>\sqrt{2}$, we have that

$$
\limsup _{N \rightarrow \infty} \sup _{u_{1}, u_{2} \in[-T, T]} \int_{I_{N}} \sqrt{\frac{N}{2 \pi}} e^{-\frac{1}{2} N r^{2}+N B(r)} \Delta_{N}\left(r, u_{1}, u_{2}\right) d r=0 .
$$

Proof. Denote by $\mathbf{M}^{(i)}=\mathbf{M}_{N-1}^{(i)}\left(r, u_{1}, u_{2}\right)$ and $\mathbf{W}^{(i)}=\mathbf{W}_{N-1}\left(u_{i}\right)$ the matrices in the numerator and denominator of (2.5), respectively. By the Cauchy-Schwarz inequality,

$$
\Delta_{N}\left(r, u_{1}, u_{2}\right) \leq \frac{\sqrt{\mathbb{E}\left\{\operatorname{det}\left(\mathbf{M}^{(1)}\right)^{2}\right\} \mathbb{E}\left\{\operatorname{det}\left(\mathbf{M}^{(2)}\right)^{2}\right\}}}{\mathbb{E}\left\{\left|\operatorname{det}\left(\mathbf{W}^{(1)}\right)\right|\right\} \mathbb{E}\left\{\left|\operatorname{det}\left(\mathbf{W}^{(2)}\right)\right|\right\}} .
$$

Hence, to prove the lemma it will be enough to show that for large $N$, uniformly in $r \in I_{N}$ and $u_{1}, u_{2} \in[-T, T]$,

$$
\frac{\mathbb{E}\left\{\operatorname{det}\left(\mathbf{M}^{(i)}\right)^{2}\right\}}{\mathbb{E}\left\{\left|\operatorname{det}\left(\mathbf{W}^{(i)}\right)\right|\right\}^{2}} \leq c N,
$$

for some constant $c$, since this would imply that

$$
\begin{aligned}
& \int_{I_{N}} \sqrt{\frac{N}{2 \pi}} e^{-\frac{1}{2} N r^{2}+N B(r)} \Delta_{N}\left(r, u_{1}, u_{2}\right) d r \\
& \leq c N e^{o_{N}(1)} \int_{\sqrt{N} I_{N}} \frac{1}{\sqrt{2 \pi}} e^{-\frac{1}{2} r^{2}} d r \leq \sqrt{\frac{2}{\pi \log N}} \frac{c}{C} e^{\left(1-\frac{1}{2} C^{2}\right) \log N+o_{N}(1)},
\end{aligned}
$$

where we used the fact that for the standard Gaussian density $\varphi(x)$, for $x \geq 0, \int_{x}^{\infty} \varphi(t) d t<\varphi(x) / x$. We prove a slightly more general result than (2.12) in the lemma below, which will be also used in Section 2.4

Lemma 8. Let $\mathbf{M}^{(i)}=\mathbf{M}_{N-1}^{(i)}\left(r, u_{1}, u_{2}\right)$ and $\mathbf{W}^{(i)}=\mathbf{W}_{N-1}\left(u_{i}\right)$ denote the matrices in the numerator and denominator of (2.5), respectively. Then for any even integer $k>1$ and $T \in\left(0, E_{\infty}\right)$, there exists a constant $c=c(k, T)>0$ such that for large $N$,

$$
\frac{\mathbb{E}\left\{\left|\operatorname{det} \mathbf{M}^{(i)}\right|^{k}\right\}}{\mathbb{E}\left\{\left|\operatorname{det} \mathbf{W}^{(i)}\right|\right\}^{k}} \leq c N^{\frac{k(k-1)}{2}},
$$

uniformly in $r \in(-1,1)$ and $u_{1}, u_{2} \in[-T, T]$.

Proof. Using (A.3),

$$
\mathbf{M}^{(i)}=\left(\begin{array}{cc}
\mathbf{G}_{N-2}^{(i)}(r)-\bar{u}_{i} I & Z^{(i)}(r) \\
\left(Z^{(i)}(r)\right)^{T} & Q^{(i)}(r)-\bar{u}_{i}+\bar{m}_{i}
\end{array}\right),
$$

where $\bar{u}_{i}:=\sqrt{\frac{N}{N-1} \frac{p}{p-1}} u_{i}$ and $\bar{m}_{i}:=\sqrt{\frac{N}{(N-1) p(p-1)}} m_{i}\left(r, u_{1}, u_{2}\right)$ and where $m_{i}\left(r, u_{1}, u_{2}\right), \mathbf{G}_{N-2}^{(i)}(r), Z^{(i)}(r)$ and $Q^{(i)}(r)$ are defined in Section $\mathrm{A}$. To lighten the notation, we henceforth omit $i$ from the super- and sub-scripts for all variables.

Let $\tilde{A}_{N-1}$ be an orthogonal matrix (measurable w.r.t. $Z(r)$ ) such that

$$
\tilde{A}_{N-1} Z(r)=\tilde{Z}(r):=(0, \ldots, 0,\|Z(r)\|),
$$

and set

$$
A_{N-1}=\left(\begin{array}{cc}
\tilde{A}_{N-1} & 0 \\
0 & 1
\end{array}\right)
$$


Then, denoting $\tilde{\mathbf{G}}_{N-2}(r):=\tilde{A}_{N-1} \mathbf{G}_{N-2}(r) \tilde{A}_{N-1}^{T}$, we have that

$$
A_{N-1} \mathbf{M} A_{N-1}^{T}=\left(\begin{array}{cc}
\tilde{\mathbf{G}}_{N-2}(r)-\bar{u} I & \tilde{Z}(r) \\
\tilde{Z}(r)^{T} & Q(r)-\bar{u}+\bar{m}
\end{array}\right) .
$$

Therefore,

$$
\begin{aligned}
\operatorname{det} \mathbf{M}=\operatorname{det}\left(A_{N-1} \mathbf{M} A_{N-1}^{T}\right) & =(Q(r)-\bar{u}+\bar{m}) \operatorname{det}\left(\tilde{\mathbf{G}}_{N-2}(r)-\bar{u} I\right) \\
& -\|Z(r)\|^{2} \operatorname{det}\left(\tilde{\mathbf{G}}_{N-3}(r)-\bar{u} I\right),
\end{aligned}
$$

where by an abuse of notation we denote by $\tilde{\mathbf{G}}_{N-3}(r)$ the $N-3 \times N-3$ upper-left submatrix of $\tilde{\mathbf{G}}_{N-2}(r)$.

Note that $\tilde{\mathbf{G}}_{N-2}(r), \tilde{Z}(r)$ and $Q(r)$ are independent, and that $\tilde{\mathbf{G}}_{N-2}(r)$ has the same law as $\mathbf{G}_{N-2}(r)$. Specifically, if we let $\hat{\mathbf{G}}_{j}$ be a $j \times j$ centered, symmetric matrix with independent elements, up to symmetry, whose off-diagonal elements have variance $1 /(N-1)$ and whose on-diagonal elements have variance $2 /(N-1)$, then, for any fixed $r, \tilde{\mathbf{G}}_{N-2}(r)$ and $\tilde{\mathbf{G}}_{N-3}(r)$ have the same law as $\hat{\mathbf{G}}_{N-2}$ and $\hat{\mathbf{G}}_{N-3}$. Using the fact that $|x+y|^{k} \leq 2^{k-1}\left(|x|^{k}+|y|^{k}\right)$,

$$
\begin{aligned}
\mathbb{E}\left\{|\operatorname{det} \mathbf{M}|^{k}\right\} & \leq 2^{k-1}\left[\mathbb{E}\left(|Q(r)-\bar{u}+\bar{m}|^{k}\right) \mathbb{E}\left(\left|\operatorname{det}\left(\hat{\mathbf{G}}_{N-2}-\bar{u} I\right)\right|^{k}\right)\right. \\
& \left.+\mathbb{E}\left(\|Z(r)\|^{2 k}\right) \mathbb{E}\left(\left|\operatorname{det}\left(\hat{\mathbf{G}}_{N-3}-\bar{u} I\right)\right|^{k}\right)\right] .
\end{aligned}
$$

Since $\mathbf{W}$ has the same law as $\hat{\mathbf{G}}_{N-1}-\bar{u} I$, to prove the lemma we need to bound the ratio of the above and

$$
\mathbb{E}\{|\operatorname{det} \mathbf{W}|\}^{k}=\mathbb{E}\left\{\left|\operatorname{det}\left(\hat{\mathbf{G}}_{N-1}-\bar{u} I\right)\right|\right\}^{k} \text {. }
$$

One can check that for large $N, r \in(-1,1)$ and $u_{i} \in\left(-E_{\infty}, E_{\infty}\right)$, the variance of each element of $Z(r)$ is bounded by $\frac{1}{N-1}$ and

$$
\begin{aligned}
\mathbb{E}\left(|Q(r)-\bar{u}+\bar{m}|^{k}\right) & \leq 3^{k} \\
\mathbb{E}\left(\|Z(r)\|^{2 k}\right) & \leq 2 .
\end{aligned}
$$

Hence, it remains to show that for large $N$ and $k>1$ even,

$$
\frac{\mathbb{E}\left(\left|\operatorname{det}\left(\hat{\mathbf{G}}_{N-2}-\bar{u} I\right)\right|^{k}\right)}{\mathbb{E}\left\{\left|\operatorname{det}\left(\hat{\mathbf{G}}_{N-1}-\bar{u} I\right)\right|\right\}^{k}}, \frac{\mathbb{E}\left(\left|\operatorname{det}\left(\hat{\mathbf{G}}_{N-3}-\bar{u} I\right)\right|^{k}\right)}{\mathbb{E}\left\{\left|\operatorname{det}\left(\hat{\mathbf{G}}_{N-1}-\bar{u} I\right)\right|\right\}^{k}} \leq C N^{\frac{k(k-1)}{2}}
$$

for some constant $C>0$, uniformly in $\bar{u} \in[-\bar{T}, \bar{T}]$ for $\bar{T}:=T \sqrt{\frac{N}{N-1} \frac{p}{p-1}}$. Note that by our assumption that $T \in\left(0, E_{\infty}\right), \bar{T}<2-\epsilon$ for some small $\epsilon>0$ and large $N$.

Toward showing (2.13), it is worthwhile to switch notation, in order to better fit existing literature. Let $\mathbf{Y}_{N-1}$ denote a GOE matrix, of dimension $N-1$, normalized so that its spectrum is supported on $[-\sqrt{2}, \sqrt{2}]$ (this is the

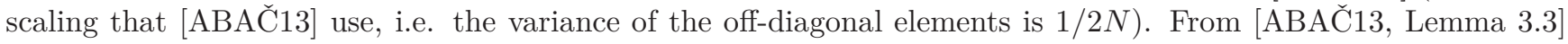
(taking $t \rightarrow 0$ with $N$ fixed) we obtain that

$$
\mathbb{E}\left(\left|\operatorname{det} \mathbf{Y}_{N-1}-x I\right|\right)=\frac{\sqrt{2} \Gamma\left(\frac{N}{2}\right) \sqrt{N(N-1)}}{(N-1)^{N / 2}} e^{(N-1) x^{2} / 2} \rho_{N}(x),
$$

where $\rho_{N}(x) d x=\mathbb{E} L_{N}(d x)$ is the expected density of states, which converges to the semicircle law at $x$ uniformly in compact subsets of $(-\sqrt{2}, \sqrt{2})$. Applying the Stirling formula, we conclude that

$$
\mathbb{E}\left(\left|\operatorname{det} \mathbf{Y}_{N-1}-x I\right|\right)=C_{1}(x) 2^{-N / 2} \sqrt{N} e^{-N / 2+(N-1) x^{2} / 2}(1+o(1)),
$$

where $C_{1}(x)$ is explicit, continuous in $x$, strictly positive on $(-\sqrt{2}, \sqrt{2})$, and the error is uniform in compact subsets of $(-\sqrt{2}, \sqrt{2})$. 
On the other hand, using [BH01, (56)] (correcting for the factor $e^{N k x^{2}} 2^{-N k / 2}$, which is missing from their formulation because they omitted the values of $f$ at the saddle point, see [BH01, (50), (54)] ), we have that

$$
\mathbb{E}\left(\left|\operatorname{det} \mathbf{Y}_{N-1}-x I\right|^{2 k}\right)=C_{2 k}(x) N^{2 k^{2}} 2^{-N k} e^{-N k+N k x^{2}}(1+o(1)),
$$

where $C_{2 k}(x)$ is an explicit constant, continuous in $x$ and independent of $N$, and strictly positive in $(-\sqrt{2}, \sqrt{2})$.

All together, we obtain that, uniformly on compact subsets of $(-\sqrt{2}, \sqrt{2})$,

$$
\frac{\mathbb{E}\left(\left|\operatorname{det} \mathbf{Y}_{N-1}-x I\right|^{2 k}\right)}{\left(\mathbb{E}\left(\left|\operatorname{det} \mathbf{Y}_{N-1}-x I\right|\right)\right)^{2 k}}=c_{2 k}(x) N^{2 k^{2}-k}(1+o(1)),
$$

where $c_{2 k}(x)$ is some positive explicit constant, continuous in $x$.

The claim (2.13) follows from (2.17) together with the fact that the ratio of the right hand side of (2.14) for $N-1$ and $N-\ell$ with $\ell$ fixed is bounded independently of $N$.

2.4. Small overlaps. In this section we treat the range of overlaps from which comes the main contribution to the ratio of the moments. Namely, we will prove the following lemma.

Lemma 9. For any $p \geq 32$ and constants $C>0$ and $T \in\left(0, E_{\infty}\right)$, the following holds, with the function B $(r)=$ $O\left(r^{3}\right)$ from Corollary 5, Setting

$$
I_{N}:=(-C \sqrt{\log N / N}, C \sqrt{\log N / N}),
$$

uniformly in $u_{1}, u_{2} \in[-T, T]$, we have that

$$
\limsup _{N \rightarrow \infty} \int_{I_{N}} \sqrt{\frac{N}{2 \pi}} e^{-\frac{1}{2} N r^{2}+N B(r)} \Delta_{N}\left(r, u_{1}, u_{2}\right) d r \leq 1 .
$$

Before we turn to the proof, we represent the matrices in the definition of $\Delta_{N}\left(r, u_{1}, u_{2}\right)($ see (2.5) as linear combinations of independent variables. Fix some $r, u_{1}$ and $u_{2}$. Let $V, V^{(1)}$ and $V^{(2)}$ be i.i.d. Gaussian vectors of length $N-2$ each with distribution

$$
N\left(0, \frac{1}{N-1} I_{N-2}\right)
$$

where $I_{N-2}$ is the identity matrix. Let $D, D^{(1)}$ and $D^{(2)}$ be i.i.d. Gaussian variables each with distribution

$$
N\left(0, \frac{2}{N-1}\right) \text {. }
$$

Recall the definition of $Z^{(i)}(r)$ and $Q^{(i)}(r)$ from Section $\mathrm{A}$

We may define all the variables above on the same probability space such that

$$
\begin{aligned}
& Z_{j}^{(i)}(r)=\sqrt{\frac{\Sigma_{Z, 11}(r)-\left|\Sigma_{Z, 12}(r)\right|}{p(p-1)}} V^{(i)}+\left(\operatorname{sgn}\left(\Sigma_{Z, 12}(r)\right)\right)^{i} \sqrt{\frac{\left|\Sigma_{Z, 12}(r)\right|}{p(p-1)}} V, \\
& Q^{(i)}(r)=\sqrt{\frac{\Sigma_{Q, 11}(r)-\left|\Sigma_{Q, 12}(r)\right|}{2 p(p-1)}} D^{(i)}+\left(\operatorname{sgn}\left(\Sigma_{Q, 12}(r)\right)\right)^{i} \sqrt{\frac{\left|\Sigma_{Q, 12}(r)\right|}{2 p(p-1)}} D .
\end{aligned}
$$

Also recall the definition of $\mathbf{G}_{N-2}^{(i)}(r)$, using the i.i.d. matrices $\overline{\mathbf{G}}_{N-2}, \overline{\mathbf{G}}_{N-2}^{(1)}$, and $\overline{\mathbf{G}}_{N-2}^{(2)}$, from Section $\mathrm{A}$ and assume they are independent of all other variables. Define

$$
\hat{\mathbf{X}}_{N-1}^{(i)}(r)=\left(\begin{array}{cc}
\mathbf{G}_{N-2}^{(i)}(r) & V^{(i)} \\
\left(V^{(i)}\right)^{T} & D^{(i)}
\end{array}\right),
$$

and note that for each $i=1,2, \hat{\mathbf{X}}_{N-1}^{(i)}(r)$ is a GOE matrix.

We have that

$$
\Delta_{N}\left(r, u_{1}, u_{2}\right)=\frac{\mathbb{E}\left\{\prod_{i=1,2}\left|\operatorname{det}\left(\mathbf{A}_{N-1}^{(i)}+\mathbf{B}_{N-1}^{(i)}\right)\right|\right\}}{\prod_{i=1,2} \mathbb{E}\left\{\left|\operatorname{det}\left(\mathbf{A}_{N-1}^{(i)}\right)\right|\right\}},
$$


where

and

$$
\mathbf{A}_{N-1}^{(i)}=\mathbf{A}_{N-1}^{(i)}\left(r, u_{i}\right)=\hat{\mathbf{X}}_{N-1}^{(i)}(0)-\sqrt{\frac{N}{N-1} \frac{p}{p-1}} u_{i} I
$$

$$
\begin{aligned}
\mathbf{B}_{N-1}^{(i)} & =\mathbf{B}_{N-1}^{(i)}\left(r, u_{1}, u_{2}\right)=\mathbf{X}_{N-1}^{(i)}(r)-\hat{\mathbf{X}}_{N-1}^{(i)}(0)+\mathbf{E}_{N-1}^{(i)}\left(r, \sqrt{N} u_{1}, \sqrt{N} u_{2}\right) \\
& =\left(\begin{array}{cc}
\mathbf{G}_{N-2}^{(i)}(r)-\mathbf{G}_{N-2}^{(i)}(0) & Z^{(i)}(r)-V^{(i)} \\
\left(Z^{(i)}(r)\right)^{T}-\left(V^{(i)}\right)^{T} & Q^{(i)}(r)-D^{(i)}+\frac{m_{i}\left(r, u_{1}, u_{2}\right)}{\sqrt{(N-1) p(p-1)}}
\end{array}\right) .
\end{aligned}
$$

To prove Lemma 9 we will need the following auxiliary results which we prove below. For any $N \times N$ symmetric matrix $\mathbf{T}$ of denote by $\lambda_{i}(\mathbf{T}), i=1, \ldots, N$, the eigenvalues of a matrix $\mathbf{T}$.

Lemma 10. (Bound on the perturbations $\mathbf{B}_{N-1}^{(i)}$ ) Fix $T>0$. For any $p \geq 3$ and $K>0$, there exists $c>0$ such that the following holds. If $t_{N}$ is a sequence such that $\liminf _{N \rightarrow \infty} t_{N}(N / \log N)^{\frac{p-3}{4}} \geq c$, then for large $N$, uniformly in $r \in I_{N}$ defined in (2.18) and $u_{1}, u_{2} \in[-T, T]$,

$$
\mathbb{P}\left\{\max _{j \leq N-1}\left|\lambda_{j}\left(\mathbf{B}_{N-1}^{(i)}\right)\right| \geq t_{N}\right\} \leq e^{-K N}
$$

Lemma 11. (Overcrowding estimate) For an $N \times N$ GOE matrix $\mathbf{G}$, for any measurable set $I \subset \mathbb{R}$,

$$
\mathbb{P}\{\mathbf{G} \text { has at least } t \text { eigenvalues in } I\} \leq \frac{10 N|I|}{t} \text {. }
$$

Lastly, we will need the following corollary of the main theorem of [Fie71.

Corollary 12. Fie71] Let $\mathbf{C}_{1}, \mathbf{C}_{2}$ be two (deterministic) real, symmetric $N \times N$ matrices and let $\lambda_{j}\left(\mathbf{C}_{i}\right)$ denote the eigenvalues of $\mathbf{C}_{i}$, ordered with non-decreasing absolute value. Suppose that the number of non-zero eigenvalues of $\mathbf{C}_{2}$ is d at most. Then, assuming $\lambda_{1}\left(\mathbf{C}_{1}\right) \neq 0$,

$$
\left|\operatorname{det}\left(\mathbf{C}_{1}+\mathbf{C}_{2}\right)\right| \leq\left|\operatorname{det}\left(\mathbf{C}_{1}\right)\right| \prod_{j=1}^{d}\left(1+\frac{\left|\lambda_{N}\left(\mathbf{C}_{2}\right)\right|}{\left|\lambda_{j}\left(\mathbf{C}_{1}\right)\right|}\right) .
$$

2.4.1. Proof of Lemma 9. Since $B(r)=O\left(N^{-\frac{3}{2}+\epsilon}\right)$ uniformly in $r \in I_{N}$ for arbitrarily small $\epsilon>0$,

$$
\lim _{N \rightarrow \infty} \int_{I_{N}} \sqrt{\frac{N}{2 \pi}} e^{-\frac{1}{2} N r^{2}+N B(r)} d r=1 .
$$

Hence, it will be enough to show that uniformly in $r \in I_{N}$ and $u_{1}, u_{2} \in[-T, T]$,

$$
\limsup _{N \rightarrow \infty} \Delta_{N}\left(r, u_{1}, u_{2}\right) \leq 1 .
$$

Fix some $\alpha, \beta, \gamma>0$ (to be determined below) and let $\mathcal{E}=\mathcal{E}_{N}\left(r, u_{1}, u_{2}\right)=\mathcal{E}_{1} \cap \mathcal{E}_{2}$ where

$$
\mathcal{E}_{1}=\bigcap_{i=1}^{2} \bigcap_{\ell=0}^{\left\lceil\log _{2} N^{\alpha}\right\rceil}\left\{\#\left\{j:\left|\lambda_{j}\left(\mathbf{A}_{N-1}^{(i)}\right)\right| \in\left[s_{\ell}, s_{\ell+1}\right)\right\} \leq N^{1+\beta} s_{\ell}\right\}, \quad \mathcal{E}_{2}=\bigcap_{i=1}^{2}\left\{\max _{j}\left|\lambda_{j}\left(\mathbf{B}_{N-1}^{(i)}\right)\right| \leq N^{-\gamma}\right\},
$$

with

$$
s_{\ell}= \begin{cases}0, & \ell=0 \\ \frac{2^{\ell}}{N^{\alpha}}, & 1 \leq \ell \leq\left\lceil\log _{2} N^{\alpha}\right\rceil \\ \infty, & \ell=\left\lceil\log _{2} N^{\alpha}\right\rceil+1\end{cases}
$$

From Corollary 12 on the event $\mathcal{E}$,

$$
\begin{aligned}
\left|\operatorname{det}\left(\mathbf{A}_{N-1}^{(i)}+\mathbf{B}_{N-1}^{(i)}\right)\right| & \leq\left|\operatorname{det}\left(\mathbf{A}_{N-1}^{(i)}\right)\right| \prod_{\ell=1}^{\left\lceil\log _{2} N^{\alpha}\right\rceil}\left(1+\frac{N^{-\gamma}}{s_{\ell}}\right)^{N^{1+\beta} s_{\ell}} \\
& \leq\left|\operatorname{det}\left(\mathbf{A}_{N-1}^{(i)}\right)\right| \exp \left\{N^{1+\beta-\gamma}\left\lceil\log _{2} N^{\alpha}\right\rceil\right\}
\end{aligned}
$$


where we used the fact that $(1+y)^{x} \leq e^{x y}$ for $x, y>0$. Hence if

$$
\gamma-\beta>1
$$

then

$$
\limsup _{N \rightarrow \infty} \frac{\mathbb{E}\left\{\prod_{i=1,2}\left|\operatorname{det}\left(\mathbf{A}_{N-1}^{(i)}+\mathbf{B}_{N-1}^{(i)}\right)\right| \mathbf{1}_{\mathcal{E}}\right\}}{\prod_{i=1,2} \mathbb{E}\left\{\left|\operatorname{det}\left(\mathbf{A}_{N-1}^{(i)}\right)\right|\right\}} \leq 1 .
$$

For a given $p \geq 3$, if we are able to choose $\alpha, \beta, \gamma>0$ such that 2.20) holds and further, uniformly in $r \in I_{N}$ and $u_{1}, u_{2} \in[-T, T]$,

$$
\limsup _{N \rightarrow \infty} \frac{\mathbb{E}\left\{\prod_{i=1,2}\left|\operatorname{det}\left(\mathbf{A}_{N-1}^{(i)}+\mathbf{B}_{N-1}^{(i)}\right)\right| \mathbf{1}_{\mathcal{E}^{c}}\right\}}{\prod_{i=1,2} \mathbb{E}\left\{\left|\operatorname{det}\left(\mathbf{A}_{N-1}^{(i)}\right)\right|\right\}}=0
$$

then (2.19) follows and the proof is completed for the same $p$.

By Hölder's inequality, for $k>2$ the numerator above is bounded by

$$
\prod_{i=1,2} \mathbb{E}\left\{\left|\operatorname{det}\left(\mathbf{A}_{N-1}^{(i)}+\mathbf{B}_{N-1}^{(i)}\right)\right|^{k}\right\}^{1 / k} \mathbb{P}\left\{\mathcal{E}^{c}\right\}^{1 / k^{\prime}}
$$

where $\frac{2}{k}+\frac{1}{k^{\prime}}=1$, i.e. $\frac{1}{k^{\prime}}=\frac{k-2}{k}$. By Lemma 8 , for some constant $c$ and even $k$,

$$
\prod_{i=1,2} \frac{\mathbb{E}\left\{\left|\operatorname{det}\left(\mathbf{A}_{N-1}^{(i)}+\mathbf{B}_{N-1}^{(i)}\right)\right|^{k}\right\}^{1 / k}}{\mathbb{E}\left\{\left|\operatorname{det}\left(\mathbf{A}_{N-1}^{(i)}\right)\right|\right\}} \leq c N^{k-1}
$$

Hence, 2.21) will follow if

$$
\limsup _{N \rightarrow \infty} \mathbb{P}\left\{\mathcal{E}^{c}\right\} N^{\frac{k(k-1)}{k-2}}=0
$$

uniformly in the stated parameters. From Lemma 11] and a union bound, uniformly in the stated parameters,

$$
N^{\frac{k(k-1)}{k-2}} \mathbb{P}\left(\mathcal{E}_{1}^{c}\right) \leq 2\left(20 N^{1-\alpha}+10 \sum_{\ell=1}^{\left\lceil\log _{2} N^{\alpha}\right\rceil-1} N^{-\beta}\right) N^{\frac{k(k-1)}{k-2}} \rightarrow_{N \rightarrow \infty} 0
$$

assuming that

$$
\min \{\alpha-1, \beta\}>\frac{k(k-1)}{k-2}
$$

Similarly, from Lemma 10, uniformly in the stated parameters,

$$
N^{\frac{k(k-1)}{k-2}} \mathbb{P}\left(\mathcal{E}_{2}^{c}\right) \rightarrow_{N \rightarrow \infty} 0
$$

whenever

$$
\frac{p-3}{4}-\gamma>0
$$

For $k=4, \alpha=7+\epsilon, \beta=6+\epsilon$ and $\gamma=7+2 \epsilon$, the inequalities (2.20), (2.24) and (2.25) hold for small $\epsilon$ and any $p \geq 32$. This completes the proof. 
2.4.2. Proof of Lemma 10, Define $\hat{\mathbf{B}}_{N-1}^{(i)}$ as the matrix obtained from $\mathbf{B}_{N-1}^{(i)}$ by replacing by 0 all the elements in the $N-2 \times N-2$ upper-left block. Recall that the $N-2 \times N-2$ upper-left block of $\mathbf{B}_{N-1}^{(i)}$ is $\mathbf{G}_{N-2}^{(i)}(r)-\mathbf{G}_{N-2}^{(i)}(0)$. Of course,

$$
\max _{j \leq N-1}\left|\lambda_{j}\left(\mathbf{B}_{N-1}^{(i)}\right)\right| \leq \max _{j \leq N-1}\left|\lambda_{j}\left(\hat{\mathbf{B}}_{N-1}^{(i)}\right)\right|+\max _{j \leq N-1}\left|\lambda_{j}\left(\mathbf{G}_{N-2}^{(i)}(r)-\mathbf{G}_{N-2}^{(i)}(0)\right)\right|
$$

Hence, by a union bound, to prove the lemma it will be sufficient to prove under the same conditions that

$$
\begin{array}{r}
\mathbb{P}\left\{\max _{j \leq N-1}\left|\lambda_{j}\left(\hat{\mathbf{B}}_{N-1}^{(i)}\right)\right| \geq t_{N}\right\} \leq e^{-K N}, \\
\mathbb{P}\left\{\max _{j \leq N-1}\left|\lambda_{j}\left(\mathbf{G}_{N-2}^{(i)}(r)-\mathbf{G}_{N-2}^{(i)}(0)\right)\right| \geq t_{N}\right\} \leq e^{-K N} .
\end{array}
$$

Assume $p \geq 3$. Recall the definition of $\mathbf{G}_{N-2}^{(i)}(r)$ from Section $\AA$, using the i.i.d. matrices $\overline{\mathbf{G}}=\overline{\mathbf{G}}_{N-2}, \overline{\mathbf{G}}^{(1)}=$ $\overline{\mathbf{G}}_{N-2}^{(1)}$, and $\overline{\mathbf{G}}^{(2)}=\overline{\mathbf{G}}_{N-2}^{(2)}$. Note that, for small $r$,

$$
\begin{aligned}
\mathbf{G}_{N-2}^{(i)}(r)-\mathbf{G}_{N-2}^{(i)}(0) & =\left(\sqrt{1-|r|^{p-2}}-1\right) \overline{\mathbf{G}}^{(i)} \pm \sqrt{|r|^{p-2}} \overline{\mathbf{G}} \\
& =\left(-\frac{1}{2}|r|^{p-2}+O\left(|r|^{2(p-2)}\right)\right) \overline{\mathbf{G}}^{(i)} \pm \sqrt{|r|^{p-2}} \overline{\mathbf{G}} .
\end{aligned}
$$

And in distribution,

$$
\mathbf{G}_{N-2}^{(i)}(r)-\mathbf{G}_{N-2}^{(i)}(0) \stackrel{d}{=} f(r) \overline{\mathbf{G}},
$$

where $f(r)=\sqrt{|r|^{p-2}}(1+o(1))$.

Hence, by BADG01, Lemma 6.3], denoting by $\lambda_{i}(\mathbf{T})$ the eigenvalues of a matrix $\mathbf{T}$,

$$
\mathbb{P}\left\{\max _{j \leq N-2}\left|\lambda_{j}\left(\mathbf{G}_{N-2}^{(i)}(r)-\mathbf{G}_{N-2}^{(i)}(0)\right)\right| \geq t\right\} \leq 2 \exp \left\{-\frac{(N-2) t^{2}}{10|r|^{p-2}}\right\},
$$

for all $r \in\left(-r_{0}, r_{0}\right)$, for some $r_{0}>0$ independent of $N$. From this bound, (2.27) follows.

Working with the expressions in Section $\mathrm{A}$, one can verify that

$$
\begin{aligned}
\mid 1-\sqrt{\frac{\Sigma_{Z, 11}(r)-\left|\Sigma_{Z, 12}(r)\right|}{p(p-1)} \mid} & \leq C|r|^{p-3}, \\
\sqrt{\frac{\left|\Sigma_{Z, 12}(r)\right|}{p(p-1)}} & \leq C|r|^{\frac{p-3}{2}}, \\
\mid 1-\sqrt{\frac{\Sigma_{Q, 11}(r)-\left|\Sigma_{Q, 12}(r)\right|}{2 p(p-1)} \mid} & \leq C|r|^{p-4}, \\
\sqrt{\frac{\left|\Sigma_{Q, 12}(r)\right|}{2 p(p-1)}} & \leq C|r|^{\frac{p-4}{2},} \\
\frac{m_{i}\left(r, u_{1}, u_{2}\right)}{\sqrt{p(p-1)}} & \leq C T|r|^{p-2},
\end{aligned}
$$

for some $C=C(p)$ and any $u_{1}, u_{2} \in[-T, T]$ and $r \in\left(-r_{0}, r_{0}\right)$ for small enough $r_{0}>0$, independent of $N$. From these bounds, for any $u_{1}, u_{2} \in[-T, T]$ and $r \in\left(-r_{0}, r_{0}\right)$, the Frobenius norm $\left\|\hat{\mathbf{B}}_{N-1}^{(i)}\right\|$ is stochastically dominated by

$$
\frac{4 C|r|^{\frac{p-3}{2}}}{\sqrt{N-1}} \chi_{N-2}+\frac{4 C|r|^{\frac{p-4}{2}}}{\sqrt{N-1}}|Y|+\frac{C T|r|^{p-2}}{\sqrt{N-1}},
$$

where $\chi_{N-2}$ is a chi variable of degree $N-2$ and $Y$ is an independent standard Gaussian variable. By a union bound, (2.27) easily follows. 
2.4.3. Proof of Lemma 11. Using Markov's inequality we obtain that

$$
\mathbb{P}\{\mathbf{G} \text { has at least } t \text { eigenvalues in } I\} \leq \frac{N \int_{I} \rho_{N}(x) d x}{t},
$$

where $\rho_{N}(x) d x=\mathbb{E} L_{N}(d x)$. By e.g. For10, (7.1.45)], $\rho_{N}(x)$ converges uniformly to the semicircle density, which is bounded above. The claim follows (with the constant 10 having no particular significance).

2.5. Proof of Theorem 1. The ratio in (1.3) is bounded from below by 1 for any $N$, so we only need to prove the matching upper bound in the limit. By Lemma 6 for some sequences of real numbers $a_{N}, b_{N} \rightarrow \min \{u, 0\}$ and $\rho_{N} \rightarrow 0$,

$$
\limsup _{N \rightarrow \infty} \frac{\mathbb{E}\left\{\left(\operatorname{Crt}_{N}((-\infty, u))\right)^{2}\right\}}{\left(\mathbb{E}\left\{\operatorname{Crt}_{N}((-\infty, u))\right\}\right)^{2}}=\limsup _{N \rightarrow \infty} \frac{\mathbb{E}\left\{\left[\operatorname{Crt}_{N}\left(\left(a_{N}, b_{N}\right),\left(-\rho_{N}, \rho_{N}\right)\right)\right]_{2}\right\}}{\left(\mathbb{E}\left\{\operatorname{Crt}_{N}\left(\left(a_{N}, b_{N}\right)\right)\right\}\right)^{2}} .
$$

By Corollary 5 , the right-hand side above is bounded by

$$
\begin{aligned}
& \limsup _{N \rightarrow \infty} \sup _{u_{1}, u_{2} \in\left(a_{N}, b_{N}\right)} \int_{\left(-\rho_{N}, \rho_{N}\right) \backslash\left(-r_{N}, r_{N}\right)} \sqrt{\frac{N}{2 \pi}} e^{-\frac{1}{2} N r^{2}+N B(r)} \Delta_{N}\left(r, u_{1}, u_{2}\right) d r \\
& +\limsup _{N \rightarrow \infty} \sup _{u_{1}, u_{2} \in\left(a_{N}, b_{N}\right)} \int_{\left(-r_{N}, r_{N}\right)} \sqrt{\frac{N}{2 \pi}} e^{-\frac{1}{2} N r^{2}+N B(r)} \Delta_{N}\left(r, u_{1}, u_{2}\right) d r,
\end{aligned}
$$

where we set $r_{N}:=C \sqrt{\log N / N}$ with some constant $C>\sqrt{2}$.

By Lemma 7 the first term above is equal to 0 and by Lemma 9 the second term is bounded above by, and therefore equal to, 1 . This completes the proof.

2.6. Proof of Corollary 2. For $E>-E_{\infty}$ and for $E \in\left(-E_{\star},-E_{\infty}\right)$, the corollary follows directly from (1.4) and Sub17a, Corollary 2], respectively. For $E=-E_{\infty}$, it follows by monotonicity of $u \mapsto \operatorname{Crt}_{N}((-\infty, u))$ and continuity of $\Theta_{p}(u)$.

\section{Appendix A. Conditional distribution of the Hessians}

In this appendix we describe, based on Sub17a, the law of the random variables in Lemma 4, which expresses the expectation of $\left[\operatorname{Crt}_{N}\left(B, I_{R}\right)\right]_{2}$. Define

$$
f_{N}(\boldsymbol{\sigma})=f_{N, p}(\boldsymbol{\sigma}):=\frac{1}{\sqrt{N}} H_{N, p}(\sqrt{N} \boldsymbol{\sigma}) .
$$

Given a (piecewise) smooth orthonormal frame field $E=\left(E_{i}\right)_{i=1}^{N-1}$ on $S^{N-1}:=\left\{\mathbf{x} \in \mathbb{R}^{N}:\|\mathbf{x}\|=1\right\}$, define

$$
\nabla f_{N}(\boldsymbol{\sigma}):=\left(E_{i} f_{N}(\boldsymbol{\sigma})\right)_{i=1}^{N-1}, \nabla^{2} f_{N}(\boldsymbol{\sigma}):=\left(E_{i} E_{j} f_{N}(\boldsymbol{\sigma})\right)_{i, j=1}^{N-1}
$$

Let $r \in(-1,1)$ and suppose that $\boldsymbol{\sigma}_{1}$ and $\boldsymbol{\sigma}_{2}$ are two points in $S^{N-1}$ such that $\boldsymbol{\sigma}_{1} \cdot \boldsymbol{\sigma}_{2}=r$. The following was shown in Sub17a, Lemma 13]. First, conditional on $\nabla f\left(\boldsymbol{\sigma}_{1}\right)=\nabla f\left(\boldsymbol{\sigma}_{2}\right)=0$, the pair $\left(f_{N}\left(\boldsymbol{\sigma}_{1}\right), f_{N}\left(\boldsymbol{\sigma}_{2}\right)\right)$ has the same distribution as $\left(U_{1}(r), U_{2}(r)\right) \sim N\left(0, \Sigma_{U}(r)\right)$, where the matrix $\Sigma_{U}(r)$ is given by (A.4) below. Second, for an appropriate choice of $E=\left(E_{i}\right)_{i=1}^{N-1}$, conditional on $f\left(\boldsymbol{\sigma}_{1}\right)=u_{1}, f\left(\boldsymbol{\sigma}_{2}\right)=u_{2}$ and $\nabla f\left(\boldsymbol{\sigma}_{1}\right)=\nabla f\left(\boldsymbol{\sigma}_{2}\right)=0$, the pair

$$
\left(\frac{\nabla^{2} f\left(\boldsymbol{\sigma}_{i}\right)}{\sqrt{(N-1) p(p-1)}}\right)_{i=1,2}
$$

has the same law as

$$
\left(\mathbf{X}_{N-1}^{(i)}(r)-\sqrt{\frac{1}{N-1} \frac{p}{p-1}} u_{i} I+\mathbf{E}_{N-1}^{(i)}\left(r, u_{1}, u_{2}\right)\right)_{i=1,2}
$$

where the $N-1 \times N-1$ matrices in (A.2) are defined as follows. The $N-1, N-1$ entry of $\mathbf{E}_{N-1}^{(i)}\left(r, u_{1}, u_{2}\right)$ is equal to $\frac{m_{i}\left(r, u_{1}, u_{2}\right)}{\sqrt{(N-1) p(p-1)}}$, (see (A.7) $)$ and all its other entries are zero. The matrices $\left(\mathbf{X}_{N-1}^{(1)}(r), \mathbf{X}_{N-1}^{(2)}(r)\right)$ are jointly 
Gaussian with block structure

$$
\mathbf{X}_{N-1}^{(i)}(r)=\left(\begin{array}{cc}
\mathbf{G}_{N-2}^{(i)}(r) & Z^{(i)}(r) \\
\left(Z^{(i)}(r)\right)^{T} & Q^{(i)}(r)
\end{array}\right)
$$

where:

(1) The random elements $\left(\mathbf{G}_{N-2}^{(1)}(r), \mathbf{G}_{N-2}^{(2)}(r)\right),\left(Z^{(1)}(r), Z^{(2)}(r)\right)$, and $\left(Q^{(1)}(r), Q^{(2)}(r)\right)$ are independent.

(2) The matrices $\mathbf{G}^{(i)}(r)=\mathbf{G}_{N-2}^{(i)}(r)$ are $N-2 \times N-2$ random matrices such that $\sqrt{\frac{N-1}{N-2}} \mathbf{G}^{(i)}(r)$ is a GOE matrix and, in distribution,

$$
\left(\begin{array}{c}
\mathbf{G}^{(1)}(r) \\
\mathbf{G}^{(2)}(r)
\end{array}\right)=\left(\begin{array}{c}
\sqrt{1-|r|^{p-2}} \overline{\mathbf{G}}^{(1)}+(\operatorname{sgn}(r))^{p} \sqrt{|r|^{p-2}} \overline{\mathbf{G}} \\
\sqrt{1-|r|^{p-2}} \overline{\mathbf{G}}^{(2)}+\sqrt{|r|^{p-2}} \overline{\mathbf{G}}
\end{array}\right),
$$

where $\overline{\mathbf{G}}=\overline{\mathbf{G}}_{N-2}, \overline{\mathbf{G}}^{(1)}=\overline{\mathbf{G}}_{N-2}^{(1)}$, and $\overline{\mathbf{G}}^{(2)}=\overline{\mathbf{G}}_{N-2}^{(2)}$ are independent and have the same law as $\mathbf{G}^{(i)}(r)$.

(3) The column vectors $Z^{(i)}(r)=\left(Z_{j}^{(i)}(r)\right)_{j=1}^{N-2}$ are jointly Gaussian, and for $j \leq N-2,\left(Z_{j}^{(1)}(r), Z_{j}^{(2)}(r)\right)$ are i.i.d. with

$$
\left(Z_{j}^{(1)}(r), Z_{j}^{(2)}(r)\right) \sim N\left(0,((N-1) p(p-1))^{-1} \cdot \Sigma_{Z}(r)\right),
$$

where $\Sigma_{Z}(r)$ is given in A.5.

(4) Lastly, $Q^{(i)}(r)$ are two Gaussian random variables with

$$
\left(Q^{(1)}(r), Q^{(2)}(r)\right) \sim N\left(0,((N-1) p(p-1))^{-1} \cdot \Sigma_{Q}(r)\right),
$$

where $\Sigma_{Q}(r)$ is given in (A.6).

To define the covariances above, we first define, for any $r \in(-1,1)$,

$$
\begin{array}{ll}
a_{1}(r)=\frac{1}{p\left(1-r^{2 p-2}\right)}, & a_{2}(r)=\frac{1}{p\left[1-\left(r^{p}-(p-1) r^{p-2}\left(1-r^{2}\right)\right)^{2}\right]}, \\
a_{3}(r)=\frac{-r^{p-1}}{p\left(1-r^{2 p-2}\right)}, & a_{4}(r)=\frac{-r^{p}+(p-1) r^{p-2}\left(1-r^{2}\right)}{p\left[1-\left(r^{p}-(p-1) r^{p-2}\left(1-r^{2}\right)\right)^{2}\right]}, \\
b_{1}(r)=-p & b_{2}(r)=-p r^{p} \\
+a_{2}(r) p^{3} r^{2 p-2}\left(1-r^{2}\right), & -a_{4}(r) p^{3} r^{2 p-2}\left(1-r^{2}\right), \\
b_{3}(r)= & b_{4}(r)=p(p-1) r^{p-2}\left(1-r^{2}\right) \\
a_{2}(r) p^{2}(p-1) r^{2 p-4}\left(1-r^{2}\right)\left[-(p-2)+p r^{2}\right], & -a_{4}(r) p^{2}(p-1) r^{2 p-4}\left(1-r^{2}\right)\left[-(p-2)+p r^{2}\right] .
\end{array}
$$
by

We define the covariance matrices $\Sigma_{U}(r)=\left(\Sigma_{U, i j}(r)\right)_{i, j=1}^{2,2}, \Sigma_{Z}(r)=\left(\Sigma_{Z, i j}(r)\right)_{i, j=1}^{2,2}$ and $\Sigma_{Q}(r)=\left(\Sigma_{Q, i j}(r)\right)_{i, j=1}^{2,2}$

$$
\Sigma_{U}(r)=-\frac{1}{p}\left(\begin{array}{ll}
b_{1}(r) & b_{2}(r) \\
b_{2}(r) & b_{1}(r)
\end{array}\right)
$$

by

$$
\begin{aligned}
& \Sigma_{Z, 11}(r)=\Sigma_{Z, 22}(r)=p(p-1)-a_{1}(r) p^{2}(p-1)^{2} r^{2 p-4}\left(1-r^{2}\right), \\
& \Sigma_{Z, 12}(r)=\Sigma_{Z, 21}(r)=p(p-1)^{2} r^{p-1}-p(p-1)(p-2) r^{p-3}+a_{3}(r) p^{2}(p-1)^{2} r^{2 p-4}\left(1-r^{2}\right),
\end{aligned}
$$


and

$$
\begin{aligned}
\Sigma_{Q, 11}(r) & =\Sigma_{Q, 22}(r)=2 p(p-1)-a_{2}(r)\left(1-r^{2}\right)\left[p(p-1) r^{p-3}\left(p r^{2}-(p-2)\right)\right]^{2} \\
& -\left(b_{3}(r), b_{4}(r)\right)\left(\Sigma_{U}(r)\right)^{-1}\left(\begin{array}{c}
b_{3}(r) \\
b_{4}(r)
\end{array}\right), \\
\Sigma_{Q, 12}(r) & =\Sigma_{Q, 21}(r)=p^{4} r^{p}-2 p(p-1)\left(p^{2}-2 p+2\right) r^{p-2}+p(p-1)(p-2)(p-3) r^{p-4} \\
& +a_{4}(r) p^{2} r^{2 p-6}\left(1-r^{2}\right)\left(p^{2} r^{2}-(p-1)(p-2)\right)^{2} \\
& -\left(b_{1}(r)+b_{3}(r), b_{2}(r)+b_{4}(r)\right)\left(\Sigma_{U}(r)\right)^{-1}\left(\begin{array}{c}
b_{2}(r)+b_{4}(r) \\
b_{1}(r)+b_{3}(r)
\end{array}\right) .
\end{aligned}
$$

Finally, we define

$$
\begin{aligned}
& m_{1}\left(r, u_{1}, u_{2}\right)=\left(b_{3}(r), b_{4}(r)\right)\left(\Sigma_{U}(r)\right)^{-1}\left(u_{1}, u_{2}\right)^{T}, \\
& m_{2}\left(r, u_{1}, u_{2}\right)=m_{1}\left(r, u_{2}, u_{1}\right) .
\end{aligned}
$$

\section{REFERENCES}

[ABA13] A. Auffinger and G. Ben Arous. Complexity of random smooth functions on the high-dimensional sphere. Ann. Probab., 41(6):4214-4247, 2013.

[ABAČ13] A. Auffinger, G. Ben Arous, and J. Černý. Random matrices and complexity of spin glasses. Comm. Pure Appl. Math., 66(2):165-201, 2013.

[AC14] A. Auffinger and W.-K. Chen. Free energy and complexity of spherical bipartite models. J. Stat. Phys., 157(1):40-59, 2014.

[AG20] A. Auffinger and J. Gold. The number of saddles of the spherical p-spin model. arXiv:2007.09269, 2020.

[AT07] R. J. Adler and J. E. Taylor. Random fields and geometry. Springer Monographs in Mathematics. Springer, New York, 2007.

[BABM21a] G. Ben Arous, P. Bourgade, and B. McKenna. Exponential growth of random determinants beyond invariance. arXiv:2105.05000, 2021.

[BABM21b] G. Ben Arous, P. Bourgade, and B. McKenna. Landscape complexity beyond invariance and the elastic manifold. arXiv:2105.05051, 2021.

[BADG01] G. Ben Arous, A. Dembo, and A. Guionnet. Aging of spherical spin glasses. Probab. Theory Related Fields, 120(1):1-67, 2001.

[BAJ21] G. Ben Arous and A. Jagannath. Shattering versus metastability in spin glasses. arXiv:2104.08299, 2021.

[BASZ20] G. Ben Arous, E. Subag, and O. Zeitouni. Geometry and temperature chaos in mixed spherical spin glasses at low temperature: the perturbative regime. Comm. Pure Appl. Math., 73(8):1732-1828, 2020.

[BH01] E. Brezin and S. Hikami. Characteristic polynomial of real symmetric random matrices. Comm. Math. Phys., 223:363-382, 2001.

[Che13] W.-K. Chen. The Aizenman-Sims-Starr scheme and Parisi formula for mixed p-spin spherical models. Electron. J. Probab., 18:no. 94, 14, 2013.

[CLR03] A. Crisanti, L. Leuzzi, and T. Rizzo. The complexity of the spherical $p$-spin spin glass model, revisited. The European Physical Journal B - Condensed Matter and Complex Systems, 36(1):129-136, 2003.

[CPS] W.-K. Chen, D. Panchenko, and E. Subag. The generalized TAP free energy. to appear in CPAM. arXiv:1812.05066.

[CPS20] W.-K. Chen, D. Panchenko, and E. Subag. The generalized TAP free energy II. Commun. Math. Phys., 2020.

[CS92] A. Crisanti and H.-J. Sommers. The spherical p-spin interaction spin glass model: the statics. Zeitschrift für Physik B Condensed Matter, 87(3):341-354, 1992.

[CS95] A. Crisanti and H.-J. Sommers. Thouless-anderson-palmer approach to the spherical p-spin spin glass model. J. Phys. I France, 5:805-813, 1995

[CS17] W.-K. Chen and A. Sen. Parisi formula, disorder chaos and fluctuation for the ground state energy in the spherical mixed p-spin models. Comm. Math. Phys., 350(1):129-173, 2017.

[Fie71] M. Fiedler. Bounds for the determinant of the sum of hermitian matrices. Proc. Amer. Math. Soc., 30:27-31, 1971.

[For10] P. Forrester. Log-gases and random matrices. Princeton University Press, 2010.

[FW07] Y. V. Fyodorov and I. Williams. Replica symmetry breaking condition exposed by random matrix calculation of landscape complexity. J. Stat. Phys., 129(5-6):1081-1116, 2007.

[Fyo04] Y. V. Fyodorov. Complexity of random energy landscapes, glass transition, and absolute value of the spectral determinant of random matrices. Phys. Rev. Lett., 92(24):240601, 4, 2004.

[JT17] A. Jagannath and I. Tobasco. Low temperature asymptotics of spherical mean field spin glasses. Comm. Math. Phys., 352(3):979-1017, 2017.

[Kiv21] P. Kivimae. The ground state energy and concentration of complexity in spherical bipartite models. arXiv:210\%.13138, 2021. 
CONCENTRATION OF THE COMPLEXITY OF SPHERICAL PURE $p$-SPIN MODELS AT ARBITRARY ENERGIES

[KTJ76] J. M. Kosterlitz, D. J. Thouless, and Raymund C. Jones. Spherical model of a spin-glass. Phys. Rev. Lett., 36:1217-1220, May 1976.

[McK21] B. McKenna. Complexity of bipartite spherical spin glasses. arXiv:2105.05043, 2021.

[SK75] D. Sherrington and S. Kirkpatrick. Solvable model of a spin glass. Phys. Rev. Lett., 35:1792-1795, 1975.

[Sub17a] E. Subag. The complexity of spherical p-spin models-A second moment approach. Ann. Probab., 45(5):3385-3450, 2017.

[Sub17b] E. Subag. The geometry of the Gibbs measure of pure spherical spin glasses. Invent. Math., 210(1):135-209, 2017.

[Sub18] E. Subag. Free energy landscapes in spherical spin glasses. arXiv:1804.10576, 2018.

[Sub21] E. Subag. The free energy of spherical pure p-spin models - computation from the TAP approach. arXiv:2101.04352, 2021.

[SZ17] E. Subag and O. Zeitouni. The extremal process of critical points of the pure $p$-spin spherical spin glass model. Probab. Theory Related Fields, 168(3-4):773-820, 2017.

[Tal06] M. Talagrand. Free energy of the spherical mean field model. Probab. Theory Related Fields, 134(3):339-382, 2006.

Eliran Subag, Department of Mathematics, Weizmann Institute of Science, Rehovot 76100, Israel.

Email address: eliran.subag@weizmann.ac.il

Ofer Zeitouni, Department of Mathematics, Weizmann Institute of Science, Rehovot 76100, Israel.

Email address: ofer.zeitouni@weizmann.ac.il 